\title{
New Quantum of the Space of the Universe and New Opportunities for the Development of Quantum Physics
}

\author{
Valentyn Alexeevich Nastasenko*
}

Professor, Department of Transport Technologies, Kherson State Maritime Academy, 141, Ushakovast. 41, 73003, Kherson, Ukraine

\section{Article Info}

\section{*Corresponding author: Valentyn Alexeevich Nastasenko Professor \\ Department of Transport Technologies \\ Kherson State Maritime Academy \\ 141, Ushakovast. 41, 73003 \\ Kherson, Ukraine \\ Tel: +38(050) 807-91-99 \\ E-mail: nastasenko2004@ukr.net}

\section{Received: March 29, 2019 \\ Accepted: April 4, 2019 \\ Published: April 10, 2019}

Citation: Nastasenko VA. New Quantum of the Space of the Universe and New Opportunities for the Development of Quantum Physics. Int J Cosmol Astron Astrophys. 2019; 1(2): 71-76.

doi: $10.18689 /$ ijcaa-1000117

Copyright: (c) 2019 The Author(s). This work is licensed under a Creative Commons Attribution 4.0 International License, which permits unrestricted use, distribution, and reproduction in any medium, provided the original work is properly cited.

Published by Madridge Publishers

\begin{abstract}
The paper provides the analysis of a number of well-known works on the substantiation of the shape and parameters of quanta of the space of the Universe, within which the dimensions of quanta are related to the wave parameters of the gravitational field. It is shown that this level of the material world is preceded by the levels of elementary particles, atoms and molecules, which are characterized by a dual state - substance and field (wave-corpuscle). On this basis, the quantum of the space of the Universe with the wave parameters of the gravitational field was associated with the graviton, as a minimal real particle of the Universe. A new rationale for the relationship of the wave parameters of the gravitational field with the wave parameters of the electromagnetic field is also suggested, which is obtained on the basis of strict physical relationships composed of fundamental physical constants: the speed c of light in vacuum, the gravitational constant $\mathrm{G}$ and Planck's constant $\mathrm{h}$. On this basis, the possibility of linking the parameters of the quantum of the space of the Universe with a single photon is shown. The simplest physical and geometric scheme of the movement of a single photon in the space of the Universe is proposed. The proposed schemes reflect the initial physical structures of the material world, which do not contradict the known laws of physics, so they can be used for further studies of the graviton and photon.
\end{abstract}

Keywords: Quantum of the space of the Universe; Shape and dimensions; Parameters of the graviton; Single photon

\section{Introduction}

The search for minimal quantum values in the Universe is associated with identifying the fundamentals of the structure of the material world. A large number of scientific works that cover the entire period of the formation of science, from ancient times to the present, have been devoted to solving this problem [1]. Previously, quantum such were atoms considered, and then the search went deeper into the area of elementary particles. The most complete study of the structure of the minimal quantum of the space of the Universe was carried out in [2], developing on the base [3] (discovery priority) and evolution of this quantum in [4] (priority new of discovery). However, the connection of these quanta with other minimal material objects of the Universe, to which the graviton and photon can be attributed, was not found. Their research is a complex scientific problem, since many of their properties and parameters are still unknown.

The goal of the work performed is to search for a possible connection between the quantum of the space of the Universe and its other material objects, in particular, the graviton and photon. 
The paper does not consider in detail the hypotheses of the physical processes of the birth and structure of the graviton and photon, obtained on the basis of the field theory and relativistic physics. The desire to take into account all the factors in these theories greatly complicates the process of finding a solution to this problem. It proposes an unconventional method based on the transition to Planck's level of the structure of the material world [5]. He suggests the opposite movement - from the simplest elements to the complex ones. Such an approach makes it possible to find a solution at the level of creating a concept with its further development in the traditional framework of quantum physics.

The scientific novelty of the work performed is the deepening of knowledge about the fundamentals of the material world on the basis of identifying the connection between the quantum of the space of the Universe and its other quantum-level objects - the graviton and photon.

\section{Of the problem analysis, setting tasks and finding the solutions.}

The minimal quantum of the space of the Universe is taken as the initial fundamentally element for further analysis, whose parameters are changed by quantum jumps to the wave parameters of the gravitational field, since they characterize real values of the oscillation frequency $v_{G}(1)$ - this Nastasenko constant and wavelength $\lambda_{G}$ (2) [6]:

$$
\begin{aligned}
& v_{G}=v_{p}=\sqrt{\frac{c^{5}}{G h}}=7.39994 \cdot 10^{42}\left(s^{-1}\right) \rightarrow 7.4 \cdot 10^{42}\left(s^{-1}\right) . \\
& \lambda_{G}=\frac{c}{v_{G}}=4.051249|432| \cdot 10^{-35}(\mathrm{~m}) .
\end{aligned}
$$

Where $G$ - gravitational constant: $G=6.67408(31) \cdot 10^{-11} \frac{\mathrm{m}^{3}}{\mathrm{~kg} \cdot \mathrm{s}^{2}}$; $c$ - speed of light in vacuum: $c=0.299792458$ (exactly) $\cdot 10^{9} \frac{\mathrm{m}}{\mathrm{s}}$;

$h$ - Planck's constant:

$h=6.626070040(81) \cdot 10^{-34} \mathrm{~J} \cdot \mathrm{s}=6.26070040(81) \cdot 10^{-34} \frac{\mathrm{kg} \cdot \mathrm{m}^{2}}{\mathrm{~s}}$.

Magnitude and accuracy of parameters $G, c, h$ is recommended CODATA [7].

To determine the wave parameters of the gravitational field in [6], the transition to Planck level of the material world was used, which is deeper than the levels of elementary particles and atoms (Figure 1). At this level, all interaction processes are reduced to the simplest elements and structures obtained on the basis of fundamental physical constants and their transformations [5].

It should be noted that the traditional approach involves the development of the field theory based on Lorentz-Maxwell equations using Einstein's theory of gravity [8-10], the theory of superstrings and other theories $[11,12]$. They provide for the complication of field and other structures material world. However, to date, no solution has been found for the wave parameters of the gravitational field by used this way.

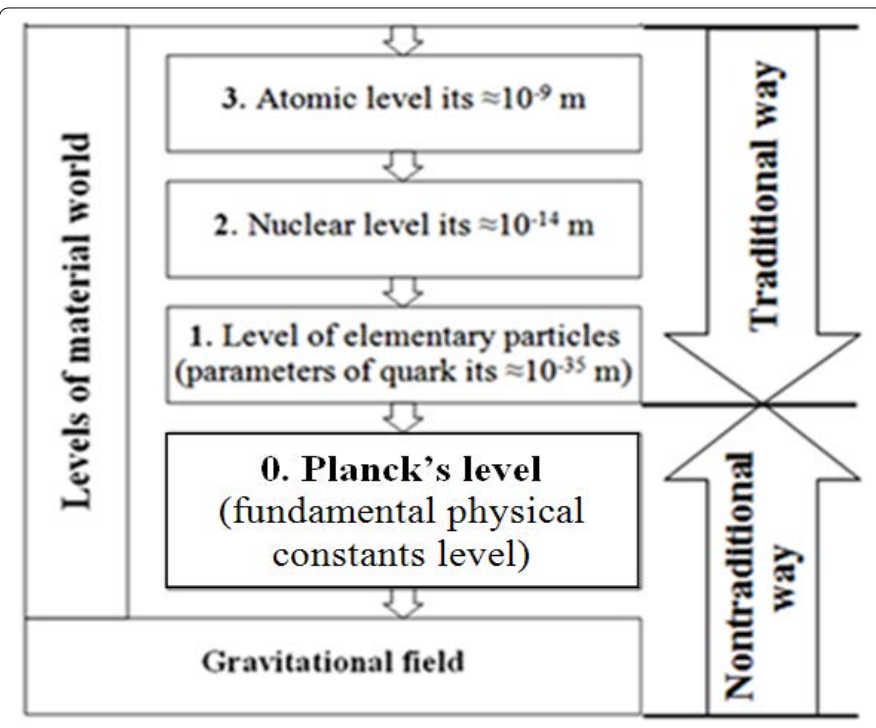

Figure 1. Quantum levels of material world (microcosm of Universe)

The proposed unconventional way [6] allowed finding a solution (1), which is physically strict. Its cannot is disprove it, since it is the only possible solution obtained on the basis of 3 fundamental physical constants $c, G$, and $h$. Therefore, the further development of the traditional way solution will lead to the same result (1). The unconventional approach at Planck's level $[5,6]$ made it possible to find the wave parameters of the gravitational field quickly and seamlessly, which confirms the expediency of its wide application in further studies.

In [3], elementary quanta of the space of the Universe were presented in the shape of trihedral figures of the original and mirror reflection forms, which together form a dipole, and 6 such dipoles form a circular quantum of the space of the Universe, with a gravitational radius inside it, which unites the dipoles (Figure 2).

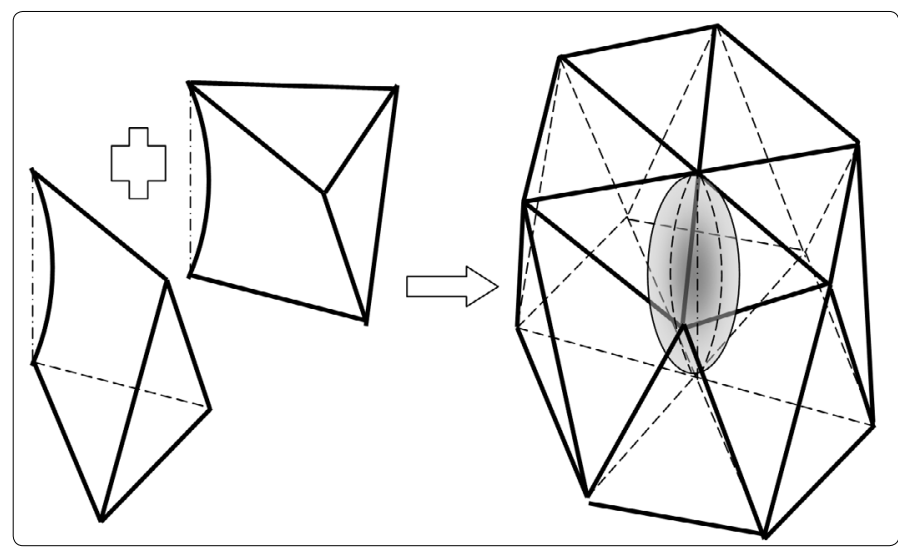

Figure 2. New image of a quantum of space of the Universe and scheme of its formation: a) elementary quantum, b) antipode, c) deformation of a square in a rhombus, $d$ ) new circular quantum

A circular quantum is a stable structure, but elementary quanta and dipoles cannot form an internal gravitational radius; therefore, they cannot exist separately. It was also justified [4] that 12 quanta form a mini-string that has a spiral shape. Gravitational radii inside these quanta form threads in the space of the Universe [4]. Mini-string has a helix coil with a pitch of 12 quanta (Figure 3). The direction of a mini-string coiling has a forward and reverse stroke. 


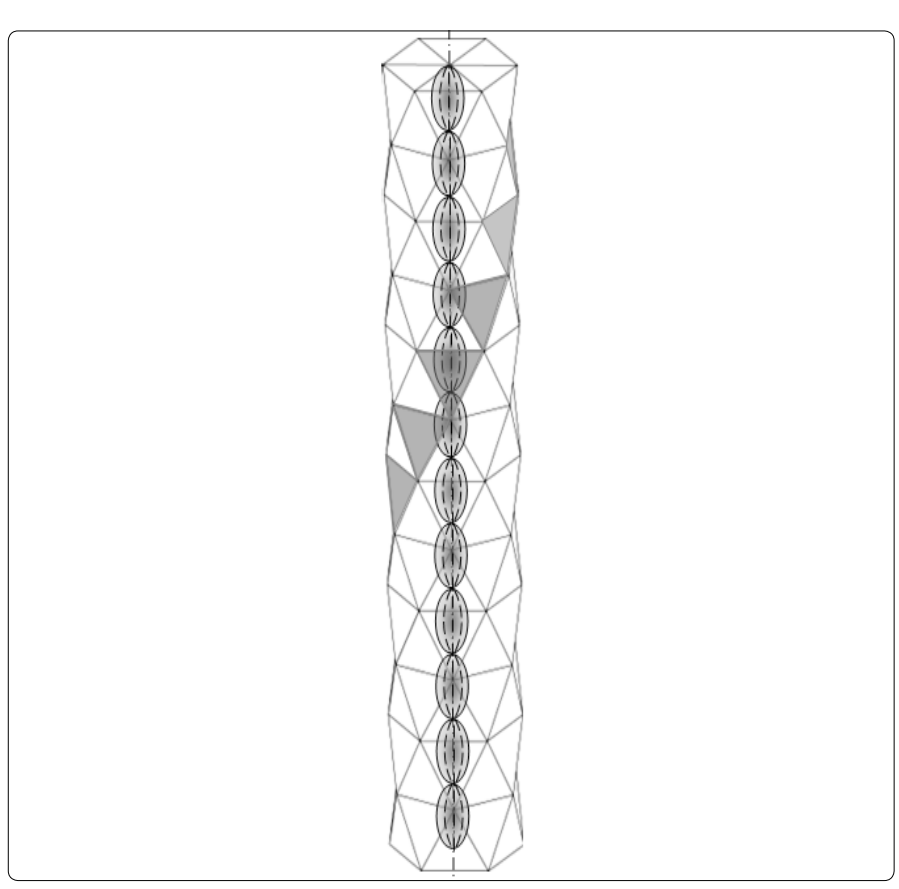

Figure 3. Formation of a complete turn of mini strings circular quanta on their 12 layers

However, the study of the minimum quantum of the space of the Universe cannot be limited to this form; it is presents the main task of further research.

\section{The substantiation for the connection of the minimal quantum of the Universe with a graviton}

The analysis of the known objects of the atomic and subatomic levels of the material world [8] showed that they are characterized by a dual state: the substances and field (corpuscle-wave). These levels of prefacing the level of quantum space of the Universe. Since quant has the wave parameters of the gravitational field [2], there is every reason to consider this quantum as a material formation, also having a corpuscle-wave structure.

It was also taken into account that on the basis of the parameters of the frequency of the gravitational field (1), the gravitational constant $\mathrm{G}$ can be obtained from addiction (3):

$$
\begin{aligned}
& G=\frac{c^{5}}{v_{G}{ }^{2} h}=\frac{\left[0.299792458 \cdot 10^{9}\left(\frac{\mathrm{m}}{\mathrm{s}}\right)\right]^{5}}{\left[7.4 \cdot 10^{42}\left(\mathrm{~s}^{-1}\right)\right]^{2} 6.626070040 \cdot 10^{-34}\left(\frac{\mathrm{kg} \cdot \mathrm{m}^{2}}{\mathrm{~s}}\right)}= \\
& =6.6739669698 \cdot 10^{-11}\left(\frac{\mathrm{m}^{3}}{\mathrm{~kg} \cdot \mathrm{s}^{2}}\right) .
\end{aligned}
$$

The second starting point is Newton's law of universal gravitation (4):

$$
F_{G}=G \frac{m_{1} m_{2}}{r^{2}}(N) \text {., }
$$

where: $m_{1} m_{2}$ - masses $(\mathrm{kg})$ of two point objects situated at the $r(m)$ - distant between them;

Since $G$ is a characteristic of the gravitational interaction that, in the framework of the law (4), manifests itself for the point real objects $m_{\gamma^{\prime}} m_{z^{\prime}}$ the quantum of the space of the Universe (Fig. 2) can be related of graviton. Its final shape has the form shown in figure 4, which outlines the following: elementary quantum and its form (a), antipode and its form (b), paired quantum - dipole (c) and circular quantum (d).

Stable limiting separating planes in elementary and circular quanta of space is no, as dislocation along them is impossible, and ribs, motion along which is possible, in the framework of Heisenberg uncertainty principle [8], have blurred configurations (spot), interpenetrating each (Figure 4).

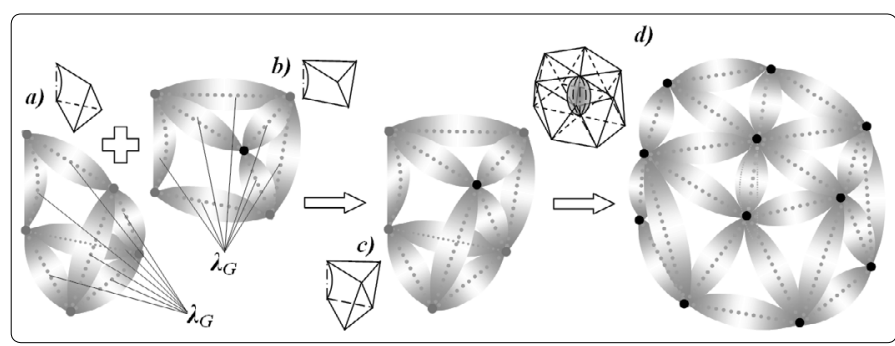

Figure 4. Formation of ribs of elementary quantum (a), antipode (b), paired quantum-dipole (c) and circular quantum (d) in the framework of Heisenberg uncertainty principle

uncertainty principle, since quantum jumps can be determined only at their initial and end points. At this level of the structure of the Universe (hereinafter referred to as Planck's level), fixed positions are material entities. The further solution of the tasks set in the work is related only to this level. At the same time, inference should be drawn that there is still void on Planck's level of the material world, but it is strictly limited to the waves of the gravitational field. Therefore, it is possible for such objects to penetrate into each other only at the similar level of their size smallness.

Currently, the graviton is known to have been theoretically grounded in the 1930-s and is presented in [13]. It has the status of a hypothetical particle, which is referred to elementary particles of the Boson family and constitutes a group of gauge bosons. As an antiparticle it is equivalent to itself, its charge is 0 $[11,12]$. It participates in gravitational interaction. In the framework of general concepts, as a particle, its mass is theoretically equal to 0 , the energy mass (upper experimental limit) is $<1.1 \cdot 10^{-29} \mathrm{eV} / \mathrm{s}^{2}$. Its quantum numbers are: baryon number is 0 , leptonic number is 0 , spin is $2 \hbar$, parity is +1 , charge parity is +1 [10-12]. These parameters were obtained on the basis of electromagnetic theories and experiments.

However, the same parameters can be obtained on the basis of the minimal quantum of the space of the Universe [4]. The dipole with the side $\lambda_{G}$ (Figure $4 c$ ) corresponds most fully to them, in which the initial quantum a) has an antipode b) (a mirror reflection), and they both taken together present a mutually balanced compound, a dipole with a neutral (zero) charge. The presence of a dipole allows the "top" and "bottom" of a graviton to strictly select. The spin $2 \hbar$, connected with the gravitational radii of the initial quantum and its antipode, also fits well into the proposed dipole scheme from the two initial elementary quanta. In the final form, 6 dipoles form a circular quantum of space (Figure 4d), while the direct and inverse coiling of its helix form also proves that a graviton has spin 2 . 
Taking into account the possibility of close packing of the space of the Universe only by structures of 6 dipoles that complete the circle, it is advisable to consider a circular quantum of the Universe as a graviton (Figure $4 \mathrm{~d}$ ), and a dipole (Figure 4c) as a component of its structure, should be considered as a finer elementary particle - a gravitino. The application of the proposed schemes (Figure 4) is acceptable for further research, since at the initial quantum level, in the framework of the principle of minimization, all objects and processes are as simple as possible. Their complication occurs in the transition from Planck's level of the material world to higher levels of elementary particles and atoms.

At this, since the birth of the Universe as a result of the Big Bang [14], the expansion of its fields could be uniform. However, due to violations of the symmetry laws occurring in the material world, in these fields the heterogeneities emerged that led to the birth of elementary particles, and furtheratoms and other material objects of the Universe originated from them, which led to the complication of the structure of the gravitational field. Since these modifications appeared at higher levels of the material world than Planck's one, they therefore go beyond the scope of the tasks of this work and are not considered in detail.

Further estimation of the energy state of the graviton is carried out within the framework of Planck's level.

The energy mass of the graviton consisting of 6 gravitino dipoles $(6 \times 2=12)$, in the period of singularity [14], composes 12 values of Planck's energy $E_{p}(5)$ :

$E_{G \max }=12 h v_{G}=5.884 \cdot 10^{10}(\mathrm{~J}) \rightarrow 12 E_{P}=12 \sqrt{\frac{h c^{5}}{G}}=5.884 \cdot 10^{10}(\mathrm{~J})$.

For the modern age of the Universe $T_{U} \approx 13.5$ billion years [14] or $\approx 4.26 \cdot 10^{17} \mathrm{~s}$, the average radius of its gravitational field is $R_{U}=c T_{U^{\prime \prime}}$ where $c$ is the speed of light in vacuum, which has gravitational waves at their propagation. The surface of the propagation of the front of the gravitational wave in it is equal to (6):

$S_{U}=\pi R_{U}^{2}=\pi\left(c T_{U}\right)^{2}=5.124 \cdot 10^{52}\left(\mathrm{~m}^{2}\right)$.

Then it is possible to calculate the number of gravitons on which the initial energy (5) dissipates. To do this, we need to determine the area of the hexagonal surface of the circular quantum of the space:

$S_{Q}=\frac{3 \sqrt{3}}{2} \lambda_{G}{ }^{2}=0.426412 \cdot 10^{-68}\left(\mathrm{~m}^{2}\right)$.

The energy of gravitons in the frontal wave decreases in proportion to the amount of gravitons contained therein and will magnitude amount to (6) which are close to 0 . However, it cannot be zero, since without the mass arising from Einstein's law on the connection of energy and mass $E=m c^{2}$, the graviton cannot exist in principle:

$E_{G Q}=\frac{12 h v_{G}}{\left(\frac{S_{U}}{S_{Q}}\right)}=0.489 \cdot 10^{-110}(J)$.

It should be taken into account that the real energetic states of the graviton in the modern Universe may differ from the value (8), but this is an average value determined within the framework of Planck's level. It magnitude can serve as a benchmark for further research.

The above schemes allow concluding that gravitons of the Universe are stable, and are stationary (do not move in its gravitational field), therefore its expansion is associated with the creation of new gravitons, with the transfer of energy to them from previous gravitons.

For other material objects of the Universe, their mass creates its own stationary gravitational field, which moves along with this material object (for example-the gravitational field of the Earth, which is the moving in orbit around the Sun, etc.). At the same time, energy indices magnitude of gravitons near these objects will be larger than those of gravitons of the Universe (8). As they move away from these objects, their energy is reduced to the merge with the general values of the Universe. Within the void between the fixed points of the graviton (Figure 4), the penetration of objects of the same level of smallness into the graviton is possible; therefore the orientation of the gravitons of the Universe and its other minimal objects may be different. However, these modifications of the material world are also beyond the bounds of Planck's level, therefore, they are not considered in detail in this work.

\section{The Substantiation for the connection of the minimal quantum of the space of the Universe with the photon}

Further analysis of dependencies (1), (3) showed that the oscillation frequency $v_{G}$ is a universal value. Therefore, on its basis other fundamental physical constants can be obtained: the speed of light c in vacuum (9) and Planck's constant $h$ (10):

$h=\frac{c^{5}}{v_{G}{ }^{2} G}=\frac{\left[0.299792458 \cdot 10^{9}\left(\frac{\mathrm{m}}{\mathrm{s}}\right)\right]^{5}}{\left[7.4 \cdot 10^{42}\left(\mathrm{~s}^{-1}\right)\right]^{2} 6.6739669698 \cdot 10^{-11}\left(\frac{\mathrm{m}^{3}}{\mathrm{~kg} \cdot \mathrm{s}^{2}}\right)}=$

$=6.626070040 \cdot 10^{-34}\left(\frac{\mathrm{kg} \cdot \mathrm{m}^{2}}{\mathrm{~s}}\right)$.

$c=\sqrt[5]{G h v_{G}^{2}}=\sqrt[5]{6.6739669698 \cdot 10^{-11}\left(\frac{\mathrm{m}^{3}}{\mathrm{~kg} \cdot \mathrm{s}^{2}}\right) 6.626070040 \cdot 10^{-34}\left(\frac{\mathrm{kg} \cdot \mathrm{m}^{2}}{\mathrm{~s}}\right)\left[7.4 \cdot 10^{42}\left(\mathrm{~s}^{-1}\right)\right]^{2}}=$

$=0.299792458 \cdot 10^{9}\left(\frac{m}{s}\right)$.

Since the constants $c$ and $h$ are characteristics of the electromagnetic field, it can therefore be argued that the oscillation frequency $v_{G}$ is the oscillation frequency of the unified field of the Universe. This allows associating the quantum of the space of the Universe with another smallest possible object-the photon. The possibilities of strict combining of the gravitational and electromagnetic fields are presented in $[15,16]$.

Unlike the graviton, the photon is recognized as a real physical elementary particle. Its real existence was confirmed experimentally in 1926 [17] on the basis of the works of Planck and Einstein [8-10]. The photon, as well as the graviton, it is 
related to the elementary particles of the Boson family and is constituted a group of gauge bosons. The number of types is 1 . It participates in electromagnetic and gravitational interactions. $\mathrm{He}$ is not identified as an antiparticle, its charge is $0\left(<10^{-35} \mathrm{e}\right)$ [18], where $e$ is the electron charge. Charge parity is 1 . Within the general idea of photon as of a particle, its mass is assumed to be 0 , theoretically it is $<10^{-22} \mathrm{eV} / \mathrm{s}^{2}[19]$. Spin is $1 \hbar$, the number of spin states is 2 , the helicity is \pm 1 , the magnetic moment equals 0 , the internal parity is uncertain [20-23]. At the same time, modern science considers the photon as a fundamental elementary particle that does not have structure and size.

In the proposed work, within the framework of the common frequency of oscillations of the gravitational and electromagnetic fields (1), (4), as well as the identification of the gravitational field waves with the parameters of the minimum quantum of the space of Universe [2], it is possible to relate the wave parameters of this quantum with a single photon wave parameters (11), (12):

$v_{\gamma}=v_{p}=\sqrt{\frac{c^{5}}{G h}}=7.39994 \cdot 10^{42}\left(s^{-1}\right) \rightarrow 7.4 \cdot 10^{42}\left(s^{-1}\right)$.

$\lambda_{\gamma}=\frac{c}{v_{\gamma}}=4.051249|432| \cdot 10^{-35}(\mathrm{~m})$.

Thus, a single photon has a structure and shape that is identical to the quantum of the space of the Universe (Figure 4). However, unlike motionless gravitons, the photon is mobile. Therefore, based on Figure 3, where a helix of 12 stationary gravitons is shown, a single photon makes a helical motion with the speed of light $c$, passing through 12 initial positions in each cycle successively (Figure 5). Stopping the photon will lead to the loss of its energy and its absorption by the graviton.

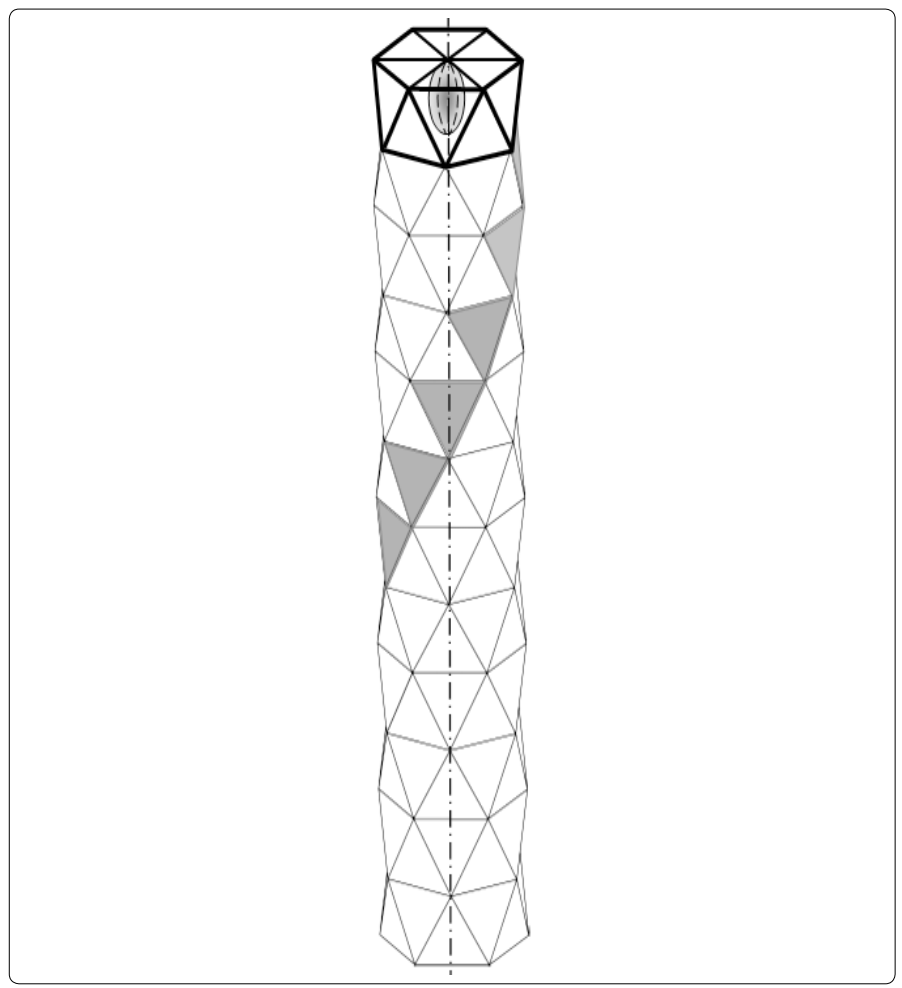

Figure 5. Single photon and its motion pattern

Herein, the upper and lower limits of the energy will be limited by the parameters (3), (8), and all energy systems that have large values present photon beams. If the energy potential of a graviton is higher than the energy potential of a photon, it prevents photons from moving through it.

Since there are no physical contradictions in all the presented schemes (Figures 2-5), it can be argued that they characterize gravitons and a single photon in its motion. At the same time, the number and structure of "colors", "oddities" and "charms", which photons and gravitons are assumed to have, can also be coordinated with these schemes.

The solution of the tasks set is obtained at the conceptual level. Their use in further research contributes to the development of physics. Other modifications of the minimal objects of the material world are beyond Planck level, therefore, in this paper they are not considered in detail.

The presentation of the structure and dimensions of a graviton and a single photon was proposed for the first time, which corresponds to the level of scientific discovery [24]. However, to justify them, experimental verification is necessary.

\section{Conclusion}

1. The quantum of the space of the Universe with the wave parameters of the gravitational field is associated with the graviton, as the minimal material particle of the Universe.

2. Within the framework of the dualism of the material world at the level of elementary particles, atoms and molecules that have a dual state of substance and field (wave-corpuscle, these same state should be considered characteristic of the graviton. At this, graviton does not exist in principle without mass (the real of its component).

3. Within the identity of the minimal quantum of the space of the Universe and the graviton, its structure should have triangular prisms with antipodes (dipoles) and comply with the principles of close packing of such quanta, which is provided by the circular arrangement of 6 dipoles around their common axis in the form of an end prism of twelve regular triangular faces.

4. Gravitons in the Universe are stable and stationary (they do not move in its gravitational field), therefore its expansion is connected with the creation of new gravitons with energy transfer from previous gravitons.

5. Within the framework of the reasonable interrelation of the wave parameters of the gravitational field with the wave parameters of the electromagnetic field, which follows from strict physical dependencies composed of fundamental physical constants: the speed $c$ of light in a vacuum of the gravitational constant $G$ and Planck's constant $h$, the quanta of the space of the Universe have a structural connection with single photons.

6. Single photons are mobile and can penetrate through gravitons with the energy potential being less than theirs. 


\section{References}

1. Vasilyeva VV, Krotovai AA, Bull DV. History of Philosophy: Textbook for universities. M.: Academic Project: 2005.

2. Nastasenko VA. Definition form and Parameters of quantum of space of the Universe. Int J CosmolAstronAstrophys. 2019 1(2): 42-45. doi: 10.18689/ ijcaa-1000111

3. Nastasenko VA. Ground of parameters minimum quantum of space of universe: Scientific Bulletin KhDMA. 2012; 1(6): 285-297.

4. Nastasenko VA. Refinement the form and parameters of quantum of space of the Universe. Int J Cosmol Astron Astrophys. 2019; 1(2): 62-66. doi: 10.18689/ijcaa-1000115

5. Nastasenko VA. Methodology for solving creative tasks high level of complexity III. 2016; 4(48): 53-59.

6. Nastasenko VA. Physical-Mathematical Method Defined of Wave Parameters Gravitation Field. Int J Eng Tech. 2019; 11(1): 62-64. doi: 10.7763/IJET.2019.V11.1124

7. CODATA Internationally recommended values of the Fundamental Physical Constants. NIST reference.

8. Alekseev DV, Bonch-Bruevich AM, Voronov-Romanov AS, et al. Physical encyclopedia hessky dictionary. Pod red. AM Prohorov (Ed.). Moskva :Sov. Encyclopedia.1983; 465: 826-33.

9. Weinberg S. Photons and Gravitons in Perturbation Theory: Derivation of Maxwell's and Einstein's Equations. Phys. Rev. 1965; 138: B988-1002. doi: 10.1103/PhysRev.138.B988

10. Rovelli C. Notes for a brief history of quantumgravity. Accessed Jue 16, 2000.

11. Dmitrievich ID, Sardanishvili GA. Gravity. LCI. 2012; 163-200.

12. Rubakov VA, Tinyakov PG. Modification of gravity at large distances and massive graviton. UFN. 2008; 178.
13. Blokhintsev DI, Halperin FM. The hypothesis of neutrinos and the law of conservation of energy. Under the banner of Marxism. 1934; 6: 147-157.

14. Silk Dj. Boljshoyjvzruv. Big Bang. 1985.

15. Nastasenko VA. Possibility of Refining the Gravitational Constant and Solving the Task of Integrating the Gravitational and Electromagnetic Fields. J Astrophys Aerospace Technol. 2018; 6(1): 155-160. doi: 10.4172/2329-6542.1000155

16. Nastasenko VA. Union of gravitational and electromagnetic fields on the basis of nontraditional principles. Electrical \& Computer Engineering An International Journal (ECIJ). 2017; 6(3-4): 19-30. doi: 10.14810/ ecij.2017.6402

17. Lewis GN. The Conservation of Photons. Nature. 1926; 118: 874-875. doi: $10.1038 / 118874 a 0$

18. Tagirov EA. Photon. Physicsofthemicroworld: a smallencyclopedia / Ch. ed. D.V. Shirkov. - Moskva: Soviet Encyclopedia. 1980; 528.

19. Paolo P, Vitor C, Leonardo G, Emanuele B, Akihiro I. Black-Hole Bombs and Photon-Mass Bounds. Physical Review Letters. 2012; 109(13): 131102. doi: 10.1103/PhysRevLett.109.131102

20. Landsberg GS. Quantumandwavepropertiesof a photon. Elementary physics text book. - 13th ed. - Moskva: Fizmatlit, - T. 3. Oscillations and waves. Optics. Atomic and nuclear physics. 2003.

21. Jennewein $T$, Achleitner $U$, Weihs $G$, Weinfurter $H$, Zeilinger $A$. A fast and compact quantum random number generator. Review of Scientific Instruments. 2000; 71: 1675-1680. doi: 10.1063/1.1150518

22. M. Tanabashietal. Review of Particle Physics. Phys. Rev. D. 2018; 98(3): 030001. doi: 10.1103/PhysRevD.98.030001

23. Scully MO, Zubairy MS. Quantum Optics. Cambridge, (CB): Cambridge University Press. 1997.

24. Civil New code Ukraine. Law of Ukraine. 2003; 142. 